\author{
e-Journal of Educational \\ Research, Assessment and \\ Evaluation
}

\title{
PERSONALIDAD Y TOMA DE DECISIONES VOCACIONALES EN UNIVERSITARIOS
}

\section{[Personality and career decision making in undergraduates]}

\author{
by/por
}

\begin{abstract}
$\underline{\text { Article record }}$
About authors

$\underline{\text { HTML format }}$
\end{abstract}

\author{
Bethencourt, José-Tomás (jbethen@ull.es) \\ Cabrera, Lidia (dcabrera@ull.es)
}

\begin{abstract}
Ficha del artículo
Sobre los autores

Formato HTML
\end{abstract}

Abstract
The relationships between personality and career decision
making in undergraduates are analyzed in this work. The
hypothesis is that efficient personality is associated with
the more mature process of career decision making. For
this hypothesis, the Questionnaire of Efficient Personality
and the Inventory of Career Factors was administered to
497 students in their final year of undergraduate school.
The collected data was put under factorial analysis, analy-
sis of differences of averages, and analysis of variance.
The results confirm that an effective personality is tied to
career decision making based as much on one's knowl-
edge of oneself as an understanding of the working world.
Keywords
Personality, Career decision making, Career development,
Undergraduates.

\section{Introducción}

La toma de decisiones vocacionales (career decision making), entendida desde el enfoque conductual-cognitivo como el proceso de resolución de un problema de elección entre alternativas socio-laborales, continúa recibiendo una abundante atención en la

\begin{abstract}
Resumen
En este trabajo se analizan las relaciones entre la personalidad y la toma de decisiones vocacionales en estudiantes universitarios. La hipótesis de partida es que la personalidad eficaz está asociada a un proceso de toma de decisiones vocacionales más maduro. Para ello se administró el Cuestionario de Personalidad Eficiente y el Inventario de Factores Vocacionales a 497 estudiantes de último y penúltimo curso de carrera. Se encontraron diferencias significativas en las dimensiones constitutivas de la decisión vocacional en grupos extremos de alta y baja personalidad eficiente. Los resultados confirman que la personalidad eficaz está vinculada a una toma de decisión vocacional más basada en el conocimiento óptimo tanto de sí mismo como del mercado laboral.
\end{abstract}

\section{Descriptores}

Personalidad, Toma de decisiones vocacionales, Desarrollo vocacional, Estudiantado universitario 
por las que discurre dicho proceso decisional (González y col., 2002; Amir, 2008).

En el presente trabajo nos centramos en el estudio de la personalidad, como uno de los tantos factores psicogénicos influyentes en la toma de decisiones vocacionales del estudiantado universitario. En las relaciones entre la personalidad y la conducta vocacional, Walsh (2004) afirma que las conclusiones de las investigaciones internacionales apuntan y sugieren que se continúe profundizando en el estudio de la influencia de los constructos de la autoeficacia y del bienestar subjetivo, rasgos propios de la personalidad eficaz, en el desarrollo y la satisfacción vocacional.

Este constructo psicológico de personalidad eficiente o personalidad eficaz, entendido como el conjunto de características personales o modos característicos que tienen las personas de enfrentarse exitosamente a su entorno, viene siendo investigado desde hace tiempo. En consecuencia, una persona caracterizada por una alta personalidad eficaz sería aquella que presenta y utiliza una alta asertividad, autoestima, capacidad de trabajo, confianza en sí mismo, estabilidad emocional, capacidad de aprender de la experiencia, conciencia comprensiva, habilidad conceptual superior, habilidad para solucionar problemas, imaginación, intuición, visión de futuro, persuasión, versatilidad, visión realista del ambiente, viveza, extraversión, flexibilidad, independencia, iniciativa, motivación de logro, optimismo, perseverancia, tolerancia a la incertidumbre, toma de riesgos y valores personales (Aciego, Domínguez y Hernández, 2005; Martín del Buey y col., 2004, 2008; Mehran, 2010; Staudinger y Bowen, 2010).

Respecto a la estructura interna de ese constructo teórico-empírico conocido como personalidad eficaz, Martín del Buey y cols. (2004) afirman que está compuesto por cuatro esferas o dimensiones del yo: fortalezas (autoconcepto y autoestima), demandas (motivación, atribución y expectativas), retos (afrontamiento de problemas y toma de decisiones) y relaciones (comunicación, empatía y asertividad).

En castellano contamos desde hace poco con un Cuestionario de Personalidad Eficiente dirigido específicamente a universitarios (Rocabert, Gómez y Descals, 2006). Está compuesto de 32 ítems, estructurado en dos factores: características no adaptativas de personalidad y personalidad eficiente. Ambos presentan una adecuada fiabilidad, con valores de consistencia interna superiores a 0.80. La estructura interna del segundo factor incluye componentes de iniciativa y optimismo, persistencia, tolerancia a la frustración, innovación y adaptabilidad, expectativas de autoeficacia, ausencia de temor ante el fracaso, autocontrol y gestión del estrés.

Para otras edades y niveles educativos, como la formación profesional, también contamos con instrumentos de contrastada bondad psicométrica para la medición de la personalidad eficaz (Dapelo y Martín del Buey, 2007; Marcone y col., 2006; Martín del Buey y col., 2008).

Asimismo, Martín del Buey y Fernández (2003) han diseñado, aplicado y evaluado un programa para el desarrollo de la personalidad eficaz, que se encuentra aún en fase de experimentación y expansión, dirigido a estudiantes de educación infantil, educación secundaria, formación profesional y educación universitaria, demostrando de momento que los cuatro componentes de la personalidad eficaz (fortalezas, demandas, retos y relaciones) pueden ser enseñados satisfactoriamente.

La influencia de la personalidad eficaz en el desarrollo académico, vocacional y social de las personas ha quedado manifiesta en diversas publicaciones (Bandura, 1997; Carbonero y Merino, 2004; Martín del Buey y col., 2004). Concretamente, nosotros hemos estudiado la relación de este constructo con el abandono de estudios universitarios (Bethencourt y cols., 2008), donde constatamos 
que las características psicológicas del alumnado son las más influyentes en la decisión de abandonar la carrera. Más concretamente, de entre todas las características psicológicas, la falta de persistencia es la que más sobresale en su incidencia en la deserción universitaria.

Sin embargo, se desconocen estudios específicos que relacionen la personalidad eficaz con la toma de decisiones vocacionales, lo cual nos motivó a realizar la presente investigación. En consecuencia, el propósito de este trabajo fue demostrar que en estudiantes universitarios de últimos cursos de carrera, la personalidad eficaz está asociada a una toma de decisión vocacional más madura. Teóricamente, se entiende por una toma de decisión vocacional madura aquella que está basada en el autoconocimiento personal, en la habilidad para aprovechar positivamente las ventajas u ocasiones presentadas en el ambiente social y académico, y menos fundamentada en razones puntuales de tipo académico-contextual (López y Rivas, 2003).

\section{Método}

\section{Objetivos}

El propósito de esta investigación fue demostrar, por un lado, que la personalidad eficaz de los estudiantes universitarios está asociada a una toma de decisiones vocacionales más madura y, por otro lado, indagar la posible existencia de diferencias en personalidad eficaz entre los cinco grupos profesionales de estudiantes universitarios considerados (cultural, biosanitario, experimental, educación-ayuda y socioeconómico). Este segundo propósito se basa en resultados científicos previos (González, Álvarez, Cabrera y Bethencourt, 2007; Martín y González, 2010), que han encontrado sistemáticamente mayor rendimiento académico y menor tasa de abandono en el grupo de estudiantes de ciencias de la salud.

En consecuencia nuestras hipótesis de partida fueron:
1) El estudiantado universitario con una alta personalidad eficaz toma decisiones vocacionales más basadas en el conocimiento de si mismo.

2) El estudiantado universitario con una alta personalidad eficaz toma decisiones vocacionales más basadas en el conocimiento del mercado laboral.

3) El estudiantado universitario con una alta personalidad eficaz toma decisiones vocacionales contando más con los recursos externos de apoyo, como familia, personas influyentes y asesoramiento.

4) Los estudiantes del grupo profesional biosanitario muestra una personalidad eficaz más alta que el alumnado de los otros grupos vocacionales.

\section{Participantes}

La población objeto de estudio ( $\mathrm{N}=5.007)$ fue el total del alumnado de último y penúltimo curso de carrera de la Universidad de La Laguna, en el conjunto de las 58 titulaciones que, exceptuados los master, oferta esta universidad. Efectuamos el muestreo en varias etapas. En la primera etapa, seleccionamos 28 titulaciones, lo que representa un $48.27 \%$ de la oferta total, de tal forma que estuvieran representados todos los grupos profesionales definidos por los estratos Cultural, Biosanitario, Educación-Ayuda, Experimental y Socioeconómico. En una segunda etapa se efectuó un muestreo aleatorio de conglomerados en los dos últimos cursos de las carreras seleccionadas. La muestra final quedó constituida por los estudiantes integrados en los conglomerados seleccionados (grupos naturales de clase) que asistían a clase en la sesión de aplicación de instrumentos.

Se aplicaron los cuestionarios a todo el alumnado presente en el aula en el momento de la recogida de datos. El total de estudiantes que cumplimentaron los instrumentos fue de 497. Siguiendo a Cardona (2002:121), para un tamaño poblacional de 5000 una 
muestra de 400 es muy adecuada, aunque decidimos dejar el total de casos obtenidos.

Las características de la muestra fueron las siguientes: 153 hombres (30.4\%) y $344 \mathrm{mu}-$ jeres (69.6\%), con una edad media de 23.56 años (DT=3.36), de los cuales 278 (56\%) cursaban estudios de Licenciatura y 219 (44\%) estudios de Diplomatura. Finalmente,
382 (77.1\%), estaban en el último curso de la carrera, y el resto, 115 (22.9\%), en el penúltimo curso, en titulaciones pertenecientes a los cinco grupos profesionales: cultural, biosanitario, experimental, educación-ayuda y socioeconómico. En la tabla 1 se muestra la distribución de la muestra atendiendo a este criterio.

Tabla 1. Distribución de los participantes por titulación y grupo profesional

\begin{tabular}{|c|c|c|c|c|c|}
\hline Titulación & $\mathbf{N}$ & $\%$ & Titulación & $\mathbf{N}$ & $\%$ \\
\hline Geografía & 18 & 3.6 & Maestro educación física & 10 & 2.0 \\
\hline Historia & 11 & 2.2 & Maestro educación infantil & 10 & 2.0 \\
\hline Bellas Artes & 24 & 4.8 & Maestro educación musical & 5 & 1.0 \\
\hline Filología Hispánica & 15 & 3.0 & Maestro educación primaria & 7 & 1.4 \\
\hline Filosofía & 11 & 2.2 & Maestro lengua extranjera & 3 & 0.6 \\
\hline Periodismo & 10 & 2.0 & Psicología & 20 & 4.0 \\
\hline Total Cultural & 89 & 18.0 & Pedagogía & 55 & 11.1 \\
\hline Enfermería & 16 & 3.2 & Psicopedagogía & 14 & 2.8 \\
\hline Logopedia & 29 & 5.8 & Total Educación-ayuda & 124 & 25.0 \\
\hline Fisioterapia & 33 & 6.6 & Derecho & 41 & 8.2 \\
\hline Biología & 12 & 2.4 & Sociología & 24 & 4.8 \\
\hline Total Biosanitario & 90 & 18.0 & Economía & 1 & 0.2 \\
\hline Matemáticas & 5 & 1.0 & Admin. y Direc.Empresas & 49 & 9.9 \\
\hline Química & 7 & 1.4 & Turismo & 16 & 3.2 \\
\hline I.T. informática gestión & 7 & 1.4 & Total Socioeconómico & 131 & 26.0 \\
\hline I.T. informática sistemas & 3 & 0.6 & \multirow{3}{*}{ Total participantes } & \multirow{3}{*}{497} & \multirow{3}{*}{100} \\
\hline Arquitectura técnica & 41 & 8.2 & & & \\
\hline Total Experimental & 63 & 13.0 & & & \\
\hline
\end{tabular}

\section{Instrumentos y procedimiento}

En la presente investigación se utilizaron los siguientes instrumentos:

1) El Cuestionario de Personalidad Eficiente de Rocabert, Gómez y Descals (2006) conformado por 32 ítems escalares con cuatro posibles valores de respuesta, donde el estudiantado ante una lista de preguntas sobre comportamientos y formas de pensar que las personas pueden manifestar habitualmente en su vida diaria, debía responder marcando la opción que mejor reflejara su caso: es exactamente mi caso, se parece bastante, es diferente a mi caso y es lo contrario. Los índices de bondad psicométrica de este instrumento han sido ya presentados en la Introducción del presente artículo. En nuestro caso, con nuestra muestra, la fiabilidad obte- nida según el alfa de Cronbach fue de 0.83. Dicho instrumento fue sometido con los datos de nuestra muestra a un nuevo análisis factorial, y los 32 ítems originales quedaron reducidos a 27, distribuidos en 4 factores que detallaremos posteriormente, a saber, Personalidad eficaz, Autocontrol y esfuerzo, Empatía y autoestima y Asertividad.

2) El Inventario de Factores Vocacionales (IFV) utilizado en el proyecto de investigación coordinado por el profesor Rivas (2005), compuesto de 22 ítems escalares, también con cuatro posibles alternativas de respuesta, donde el estudiantado universitario debía valorar una lista de enunciados según creyera que habían influido en la elección de la carrera que cursaba actualmente, seleccionando la opción que mejor reflejara su caso: mucha influencia, bastante, poca y 
ninguna influencia. El índice alfa de Cronbach obtenido en nuestra muestra para el IFV fue de 0.79. Del mismo modo que con el anterior instrumento, este también fue sometido a análisis factorial cuyos resultados mostramos con posterioridad. De los 22 ítems originales, en nuestra investigación quedaron reducidos a 21, distribuidos en 7 factores: Autoconocimiento y autoconfianza, Ambiente familiar, Facilidad de los estudios, Apoyo externo, Salidas profesionales, Fortuismo y Conocimiento laboral y oportunismo formativo.

Los instrumentos fueron aplicados colectivamente en los grupos naturales de clase seleccionados por cinco alumnas de Psicopedagogía debidamente formadas para ello ${ }^{[i]}$. La fecha de recogida de los datos fue durante los meses de marzo a mayo del curso académico. El estudiantado encuestado debía responder solamente a una de las cuatro opciones de respuesta para cada ítem de los dos instrumentos considerados. Los datos obtenidos fueron sometidos a procesamiento estadístico con el programa SPSS versión 17.0 para Windows.

\section{Análisis y Resultados}

Presentamos en primer lugar los análisis factoriales efectuados en una primera etapa de los análisis para reducir los datos originales y comprobar si la estructura factorial original se replica en nuestra muestra. En la segunda fase del análisis, aplicamos los constructos identificados en dicha estructura para responder a las preguntas formuladas en nuestra investigación.

$1^{a}$ Fase: factorialización de los Instrumentos

(a) Del Cuestionario de Personalidad Eficiente
Los 32 ítems del instrumento de personalidad fueron sometidos a análisis de componentes principales con rotación varimax. Se generaron 4 factores que se describen en la tabla 2. Decidimos adoptar esta estructura, a diferencia de la estructura bifactorial obtenida por los autores de la prueba, porque la amplitud y contenido de sus constructos se adapta mejor a nuestros propósitos. Consideramos que la estructura bifactorial de la versión de las autoras (Rocabert, Gómez y Descals, 2006) reduce excesivamente la diversidad de aspectos y componentes de la personalidad eficaz y, por tanto, ésta no resulta adecuadamente reflejada.

El factor 1 que hemos denominado Personalidad eficaz está conformado por los ítems que reflejan en los universitarios iniciativa y optimismo, persistencia, tolerancia a la frustración, adaptabilidad, autoeficacia, y finalmente, ausencia de temor al fracaso. Este factor es el que presenta el más alto porcentaje de explicación de la varianza (18.36\%) y el que mayor número de ítems agrupa, lo cual le da una naturaleza y significación psicológica muy rica y diversa. El factor 2, $A u$ tocontrol y esfuerzo, agrupa cinco ítems que señalan características de personalidad centradas en la capacidad para el control de si mismo en situaciones tanto de interacción social como de desempeño de tareas. El factor 3, Empatía y autoestima, recoge tan solo dos ítems que apuntan hacia la capacidad de los universitarios para practicar la empatía y hacer atribuciones externas de los fracasos, de modo que no dañe la autoestima positiva. Finalmente, el factor 4 Asertividad también incluye dos ítems que denotan la capacidad para el establecimiento de relaciones interpersonales positivas, caracterizadas por la evitación de conflictos innecesarios, por la expresión segura de los propios sentimientos y por la defensa respetuosa de los deseos y necesidades de uno mismo. 
Tabla 2. Estructura factorial del Cuestionario de Personalidad Eficiente

\begin{tabular}{|c|c|c|}
\hline Factor & Items & $\begin{array}{c}\text { Satura- } \\
\text { ción }\end{array}$ \\
\hline \multirow[b]{2}{*}{$\begin{array}{l}1 \text { Personalidad } \\
\text { Eficaz } \\
18.36 \%\end{array}$} & $\begin{array}{l}\text { Ítems positivos } \\
\text { - Me siento segur@ de mí mism@ para conseguir el trabajo que pienso desempe- } \\
\text { ñar. } \\
\text { - Cuando se agravan los problemas, saco nuevas fuerzas. } \\
\text { - Puedo alcanzar los objetivos profesionales que me he propuesto. } \\
\text { - Cuando tomo decisiones, tiendo a confiar en mis propias ideas y formas de hacer } \\
\text { las cosas. } \\
\text { - Cuando tengo que afrontar algo o pasar por alguna situación no agradable, pre- } \\
\text { fiero actuar en lugar de quedarme dándole vueltas y quejándome de la situación. } \\
\text { - Cuando me propongo algo, lo sigo intentando aunque al principio no lo consiga. } \\
\text { - Cuando me enfrento a un reto difícil, suelo centrarme en los aspectos positivos } \\
\text { de la situación y evito pensar en el posible fracaso. } \\
\text { - Cuando he de expresar o defender lo que pienso y mi planteamiento choca con el } \\
\text { de otras personas, vacilo y opto por no decir nada. }\end{array}$ & $\begin{array}{l}.60 \\
.57 \\
.51 \\
.51 \\
.47 \\
.47 \\
.41\end{array}$ \\
\hline & $\begin{array}{l}\text { Ítems negativos } \\
\text { - Cuando alguien me hace una crítica fuerte, pienso que no valgo nada, que todo } \\
\text { lo hago mal. } \\
\text { - Rechazo los retos difíciles para evitar la decepción que me produce el no conse- } \\
\text { guirlos. } \\
\text { - Me veo asaltad@ por el miedo, la ansiedad y un profundo malestar personal, } \\
\text { cuando tengo que afrontar algún cambio. } \\
\text { - Pienso que soy un inútil. } \\
\text { - El acabar los estudios me produce ansiedad y temor por si no soy capaz de enca- } \\
\text { jar en el trabajo. } \\
\text { - Me siento mal cuando pienso en tener que buscar trabajo. } \\
\text { - Temo no alcanzar las metas que me he propuesto. } \\
\text { - Si no consigo el trabajo que quiero seré un fracasad@. } \\
\text { - Me siento mal porque cambio demasiado de planes. } \\
\text { - No me merece la pena esforzarme, haga lo que haga me va a ser muy difícil } \\
\text { trabajar en lo que me gusta. }\end{array}$ & $\begin{array}{l} \\
-.60 \\
-.60 \\
-.55 \\
-.54 \\
-.53 \\
-.53 \\
-.50 \\
-.46 \\
-.42\end{array}$ \\
\hline $\begin{array}{l}2 \text { Autocontrol y } \\
\text { esfuerzo } \\
7.82 \%\end{array}$ & $\begin{array}{l}\text { - Cuando me relaciono con alguien, procuro captar sus gestos, tono de voz, etc., } \\
\text { para anticipar y comprender mejor la situación. } \\
\text { - Cuando hablo con otras personas suelo hacerme una idea bastante adecuada de } \\
\text { cómo reaccionarán. } \\
\text { - Cuando tengo que hacer algo importante, suelo esforzarme y mantener la activi- } \\
\text { dad al máximo. } \\
\text { - Cuando me enfrento a una tarea, conozco cuales son mis recursos, mis capacida- } \\
\text { des y mis limitaciones. } \\
\text { - Si surgen contratiempos que me impiden conseguir los objetivos en los plazos } \\
\text { previstos, analizo si es por acontecimientos ajenos a mi voluntad y trato de con- } \\
\text { trolarlos para lograr lo que me he propuesto. }\end{array}$ & $\begin{array}{l}.53 \\
.52 \\
.45 \\
.43 \\
.41\end{array}$ \\
\hline $\begin{array}{l}3 \text { Empatía y } \\
\text { autoestima } \\
4.95 \%\end{array}$ & $\begin{array}{l}\text { - Cuando un amigo me cuenta un problema me cuesta ponerme en su lugar. } \\
\text { - Si realizo un examen y obtengo mal resultado, no dudo de mi competencia gene- } \\
\text { ral. }\end{array}$ & $\begin{array}{l}-.53 \\
.45\end{array}$ \\
\hline $\begin{array}{l}4 \text { Asertividad } \\
4.08 \%\end{array}$ & $\begin{array}{l}\text { - Cuando alguien me "hace una mala pasada” prefiero callarme y tenerlo en cuen- } \\
\text { ta para situaciones futuras. } \\
\text { - Cuando alguien procede mal conmigo le hago saber como me siento, de modo } \\
\text { directo, sin avasallarlo y reconociéndole el derecho a explicarse. }\end{array}$ & .52 \\
\hline & $\begin{array}{l}\text { Total varianza explicada } \\
\end{array}$ & $35.21 \%$ \\
\hline
\end{tabular}



les

(b) Del Inventario de Factores Vocaciona-

Los 22 ítems del instrumento de toma de decisiones vocacionales fueron igualmente sometidos a un análisis de componentes principales, obteniendo una solución facto- rial de 7 factores que se describe en la tabla 3. Como en el caso del cuestionario de personalidad, adoptamos nuestra estructura factorial porque el factor añadido representa una subdimensión altamente relevante en el constructo de toma de decisiones vocacionales.

Tabla 3. Estructura factorial del Inventario de Factores Vocacionales

\begin{tabular}{|c|c|c|}
\hline Factor & Items & $\begin{array}{c}\text { Satura- } \\
\text { ción }\end{array}$ \\
\hline $\begin{array}{l}1 \text { Autoconoci- } \\
\text { miento y autocon- } \\
\text { fianza } \\
\quad 13.30 \%\end{array}$ & $\begin{array}{l}\text { El conocimiento que tengo sobre mí mismo, mis capacidades y mis posibilida- } \\
\text { des de éxito. } \\
\text { La confianza que tengo en desarrollar con éxito estos estudios. } \\
\text { Mi constancia, capacidad de trabajo y sacrificio. } \\
\text { Estar “al tanto” de las posibilidades favorables que se han cruzado en mi cami- } \\
\text { no. } \\
\text { Emplear el tiempo, el horario (trabajo y ocio), de manera adecuada para mí. }\end{array}$ & $\begin{array}{l}.82 \\
.81 \\
.69 \\
.62 \\
.44\end{array}$ \\
\hline $\begin{array}{l}2 \text { Ambiente } \\
\text { familiar } \\
\qquad 12.13 \%\end{array}$ & $\begin{array}{l}\text { La influencia favorable de mi madre (deseos, gustos, éxitos, consejos, profe- } \\
\text { sión,...). } \\
\text { La influencia favorable de mi padre (deseos, gustos, éxitos, consejos, profe- } \\
\text { sión,...). } \\
\text { El ambiente favorable de mi casa hacia estos estudios. } \\
\text { Las posibilidades y recursos económicos de mi familia (muchas / pocas). }\end{array}$ & $\begin{array}{l}.84 \\
.80 \\
.80 \\
.59\end{array}$ \\
\hline $\begin{array}{l}3 \text { Facilidad de los } \\
\text { estudios } \\
\quad 9.63 \%\end{array}$ & $\begin{array}{l}\text { La duración de los estudios. } \\
\text { La facilidad de los estudios. } \\
\text { El poder estudiar cerca de mi residencia sin tener que desplazarme. }\end{array}$ & $\begin{array}{l}.84 \\
.82 \\
.58\end{array}$ \\
\hline $\begin{array}{l}4 \text { Apoyo externo } \\
8.89 \%\end{array}$ & $\begin{array}{l}\text { El apoyo, estímulo y ayuda de algún/a profesor/a concreto. } \\
\text { El asesoramiento vocacional recibido o buscado para elegir estos estudios o } \\
\text { especialidad. } \\
\text { Haber conocido a una persona importante para mí, de la carrera o profesión que } \\
\text { he elegido } \\
\text { Mi preparación escolar anterior, las materias que me iban bien, etc. }\end{array}$ & $\begin{array}{l}.84 \\
.63 \\
.60 \\
.59\end{array}$ \\
\hline $\begin{array}{l}\text { 5 Salidas profe- } \\
\text { sionales } \\
\quad 6.71 \%\end{array}$ & Las salidas profesionales de lo que he decidido estudiar. & .79 \\
\hline $\begin{array}{r}6 \text { Fortuismo } \\
6.67 \%\end{array}$ & $\begin{array}{l}\text { La suerte o la casualidad. } \\
\text { Mis gustos y preferencias por las materias que se tratan en la carrera. }\end{array}$ & $\begin{array}{r}.74 \\
-.51\end{array}$ \\
\hline $\begin{array}{l}7 \text { Conocimiento } \\
\text { laboral y oportu- } \\
\text { nismo formativo } \\
\quad 6.03 \%\end{array}$ & $\begin{array}{l}\text { El conocimiento que ya tenía sobre el mundo del trabajo al que me dirijo. } \\
\text { Aprovechar las oportunidades de formación que se me han presentado (cursos, } \\
\text { conferencias, etc.). }\end{array}$ & $\begin{array}{l}.77 \\
.49\end{array}$ \\
\hline & Total varianza explicada & $63.37 \%$ \\
\hline
\end{tabular}

El factor 1, que hemos denominado Autoconocimiento y autoconfianza, está conformado por cinco ítems que reflejan una toma de decisiones vocacionales en los universitarios basada tanto en el buen conocimiento de si mismo como en la confianza en las propias capacidades para alcanzar las metas establecidas. El factor 2 Ambiente familiar agrupa cuatro ítems que señalan la influencia de los padres y de las circunstancias familiares en la toma de decisión vocacional. El tercero, Facilidad de los estudios, recoge tres ítems que apuntan hacia la consideración de la dificultad, duración y cercanía geográfica de las carreras universitarias en el proceso de elección vocacional. El factor cuarto, Apoyo externo, incluye cuatro ítems que denotan la 
importancia del profesorado, del asesoramiento vocacional, de los modelos profesionales y del propio historial académicoescolar en el proceso de decisión. El quinto factor de Salidas profesionales recoge tan solo un ítem muy distintivo, que refleja la gran importancia en los universitarios de las salidas laborales y oportunidades de empleo de las diferentes carreras, a la hora de ser tenidas en cuenta para su elección. El factor 6 Fortuismo reúne dos ítems uno de ellos con signo negativo, apuntado hacia la consideración del azar, la casualidad, o la suerte como razones de elección vocacional y a la omisión o desconsideración de los propios intereses o preferencias vocacionales. Finalmente, el factor 7 Conocimiento laboral y oportunismo formativo incluye también dos ítems que denotan la importancia de conocer bien el mercado laboral y de saber aprovechar las oportunidades de formación que se presenten.

\section{$2^{a}$ Fase: Personalidad y toma de decisio- nes vocacionales}

A partir de los resultados de ambas estructuras factoriales, efectuamos un sumatorio de ítems para obtener la puntuación final de los participantes en cada factor de los dos instrumentos. A partir de estas puntuaciones se calculó la media y desviación típica de la muestra en cada factor, lo que nos permitió identificar grupos con puntuaciones altas o bajas en cada una de las dimensiones obtenidas por nosotros con el Cuestionario de Personalidad Eficiente. Para ello, se identificaron los estudiantes situados en el intervalo superior e inferior, obteniendo dos grupos de sujetos extremos: en el grupo “alto", los estudiantes con puntuaciones en dichos factores iguales o superiores a media $+1 D T$; en el grupo "bajo", los estudiantes con puntuaciones iguales o inferiores a media-1DT. Los estudiantes con puntuaciones intermedias se desestimaron para esta parte de los análisis, aunque se retomaron para el contraste por grupos profesionales. Esto se efectuó para cada uno de los factores de personalidad por separado, por lo que el tamaño de los dos grupos extremos difiere según el factor de personalidad considerado (lógicamente, esto afecta a la cifra de grados de libertad, que varía para los contrastes de cada factor, como se observará en las tablas correspondientes).

Con la finalidad de determinar si las diversas dimensiones de la personalidad eficiente afectan de forma significativa la toma de decisiones vocacionales, se efectuaron contrastes de medias entre los dos grupos extremos obtenidos en cada factor de personalidad. Como variables dependientes se emplearon las puntuaciones globales en los factores que obtuvimos a partir del instrumento de Toma de Decisiones. Posteriormente, con la finalidad de comprobar si había diferencias en los factores de personalidad entre los estudiantes pertenecientes a cada uno de los grupos profesionales, según titulación cursada, llevamos a cabo un ANOVA simple, esta vez aplicado a la totalidad de los casos de la muestra.

Los resultados que mostramos a continuación siguen la siguiente secuencia, en primer lugar, presentamos las diferencias en toma de decisiones vocacionales entre los grupos altos y bajos de cada factor de personalidad. En segundo lugar, mostramos las diferencias en los factores de personalidad eficiente entre los cinco grupos profesionales.

A) Toma de decisiones vocacionales y características de la Personalidad Eficiente

\section{Decisiones vocacionales y personalidad eficaz}

Los grupos extremos detectados para el factor 1 (alta/baja personalidad eficaz) presentan diversas diferencias significativas en sus decisiones vocacionales. En la tabla 4 puede observarse que los estudiantes con personalidad eficaz se diferencian por tomar decisiones vocacionales basadas en el autoconocimiento y la autoconfianza, en el conocimiento del mercado laboral y el aprovechamiento positivo de las oportunidades de formación que se les presentan. Asimismo, los estudiantes con personalidad eficaz con- 
fían menos en el azar, la suerte o la casualidad a la hora de tomar sus decisiones vocacionales, también tienden a estar menos influenciados por sus familias o por apoyos externos, al realizar sus elecciones vocacionales maduras, aunque en estos dos factores el nivel de significación del contraste se sitúa ligeramente por encima de 0.05. Ambos grupos, en cambio, a la hora de tomar sus decisiones coinciden en otorgar similar importancia a las salidas laborales y a la facilidad de las carreras universitarias.

Tabla 4. Diferencias de medias en decisiones vocacionales según personalidad eficaz

\begin{tabular}{|l|c|c|c|c|}
\hline Factores vocacionales & $\begin{array}{c}\text { Personalidad eficaz alta } \\
\text { M (DT) }\end{array}$ & $\begin{array}{c}\text { Personalidad } \\
\text { eficaz baja } \\
\text { M (DT) }\end{array}$ & Prob. & t (gl=196) \\
\hline F1. Autoconocimiento & $14.52(3.06)$ & $11.86(3.09)$ & .000 & -5.944 \\
\hline F2. Ambiente familiar & $8.26(3.04)$ & $9.10(3.16)$ & .062 & 1.876 \\
\hline F3. Facilidad de los estudios & $4.90(1.83)$ & $5.08(1.94)$ & .506 & 0.667 \\
\hline F4. Apoyo externo & $7.18(2.72)$ & $7.96(2.87)$ & .054 & 1.935 \\
\hline F5. Salidas profesionales & $2.42(1.10)$ & $2.57(0.91)$ & .307 & 1.030 \\
\hline F6. Fortuismo & $3.32(1.31)$ & $4.36(1.48)$ & .000 & 5.151 \\
\hline F7. Conoc. laboral y oport. Formativo & $4.90(1.55)$ & $4.25(1.38)$ & .003 & -3.027 \\
\hline
\end{tabular}

\section{Decisiones vocacionales y autocontrol y} esfuerzo

En la tabla 5 se puede observar que los estudiantes con alta capacidad de autocontrol, de esfuerzo y que saben dirigir bien su voluntad hacia lo que pretenden, se caracterizan por tomar decisiones vocacionales basadas en el conocimiento tanto de si mismos como del mercado laboral, e igualmente sus decisiones vocacionales están poco guiadas por el azar o la casualidad. En el resto de factores vocacionales no presenta diferencias significativas con el grupo de autocontrol y esfuerzo bajo.

Tabla 5. Diferencias de medias en decisiones vocacionales según autocontrol y esfuerzo

\begin{tabular}{|l|c|c|c|c|}
\hline Factores vocacionales & $\begin{array}{c}\text { Autocontrol y es- } \\
\text { fuerzo alto } \\
\text { M (DT) }\end{array}$ & $\begin{array}{c}\text { Autocontrol y } \\
\text { esfuerzo bajo } \\
\text { M (DT) }\end{array}$ & Prob. & $\begin{array}{c}\text { t } \\
\text { (gl=108) }\end{array}$ \\
\hline F1. Autoconocimiento & $15.01(2.80)$ & $12.17(3.06)$ & .000 & -4.882 \\
\hline F2. Ambiente familiar & $9.23(3.46)$ & $8.39(2.93)$ & .194 & -1.306 \\
\hline F3. Facilidad de los estudios & $5.33(2.16)$ & $4.75(1.62)$ & .140 & -1.488 \\
\hline F4. Apoyo externo & $8.47(3.15)$ & $7.52(2.71)$ & .110 & -1.612 \\
\hline F5. Salidas profesionales & $2.36(0.99)$ & $2.61(1.01)$ & .202 & 1.283 \\
\hline F6. Fortuismo & $3.25(1.44)$ & $3.90(1.65)$ & .035 & 2.138 \\
\hline F7. Conoc. laboral y oport. Formativo & $5.14(1.64)$ & $3.97(1.07)$ & .000 & -4.111 \\
\hline
\end{tabular}

\section{Decisiones vocacionales y empatía y au- toestima}

En la tabla 6 se constata que el estudiantado universitario caracterizado por alta empatía y autoestima toma sus decisiones vocacionales atendiendo a diversos aspectos externos y sociogénicos, como son la facilidad de los estudios, el apoyo externo recibido y el ambiente familiar favorable. Este resultado contrasta con el obtenido en el factor de personalidad eficaz. En este caso, los sujetos con alta empatía y autoestima hacen elecciones vocacionales más dependientes del entorno social y académico y menos dependientes de aspectos internos, psicogénicos o centrados en sí mismos. 
Tabla 6. Diferencias de medias en decisiones vocacionales según empatía y autoestima

\begin{tabular}{|l|c|c|c|c|}
\hline Factores vocacionales & $\begin{array}{c}\text { Empatía y autoesti- } \\
\text { ma alta } \\
\text { M (DT) }\end{array}$ & $\begin{array}{c}\text { Empatía y auto- } \\
\text { estima baja } \\
\text { M (DT) }\end{array}$ & Prob. & $\begin{array}{c}\text { t } \\
\text { (gl=181) }\end{array}$ \\
\hline F1. Autoconocimiento & $13.67(3.15)$ & $12.98(3.06)$ & .139 & -1.488 \\
\hline F2. Ambiente familiar & $9.37(3.08)$ & $8.38(3.40)$ & .042 & -2.043 \\
\hline F3. Facilidad de los estudios & $5.82(1.95)$ & $4.67(1.67)$ & .000 & -4.196 \\
\hline F4. Apoyo externo & $8.23(2.90)$ & $6.90(2.43)$ & .001 & -3.365 \\
\hline F5. Salidas profesionales & $2.55(1.08)$ & $2.40(0.98$ & .345 & -0.947 \\
\hline F6. Fortuismo & $3.78(1.44)$ & $3.71(1.44)$ & .740 & -0.332 \\
\hline F7. Conoc. laboral y oport. Formativo & $4.75(1.46)$ & $4.45(1.44)$ & .174 & -1.364 \\
\hline
\end{tabular}

\section{Decisiones vocacionales y asertividad}

Finalmente, en cuanto a las diferencias decisionales según el factor Asertividad, en la tabla 7 se puede observar que, en general, el estudiantado universitario con alta asertividad toma decisiones vocacionales de un mo- do muy similar al grupo de baja asertividad. Este resultado se aplica por igual a los diversos factores de decisión considerados excepto por lo que respecta a la influencia del ambiente familiar, que es significativamente menos importante para el estudiantado de alta asertividad a la hora de elegir carrera.

Tabla 7. Diferencias de medias en decisiones vocacionales según asertividad

\begin{tabular}{|l|c|c|c|c|}
\hline Factores vocacionales & $\begin{array}{c}\text { Asertividad alta } \\
\text { M (DT) }\end{array}$ & $\begin{array}{c}\text { Asertividad baja } \\
\text { M (DT) }\end{array}$ & Prob. & $\begin{array}{c}\text { t } \\
\text { (gl= 140) }\end{array}$ \\
\hline F1. Autoconocimiento & $13.88(2.71)$ & $13.18(2.64)$ & .199 & -1.291 \\
\hline F2. Ambiente familiar & $8.10(2.83)$ & $9.56(3.52)$ & .016 & 2.427 \\
\hline F3. Facilidad de los estudios & $5.02(1.81)$ & $5.62(2.31)$ & .127 & 1.535 \\
\hline F4. Apoyo externo & $7.35(2.54)$ & $8.34(3.25)$ & .122 & 1,803 \\
\hline F5. Salidas profesionales & $2.46(1.02)$ & $2.43(1.01)$ & .899 & -0.127 \\
\hline F6. Fortuismo & $3.49(1.36)$ & $3.84(1.43)$ & .210 & 1.259 \\
\hline F7. Con. laboral y oport. formativo & $4.56(1.46)$ & $4.31(1.71)$ & .414 & -0.819 \\
\hline
\end{tabular}

B) Diferencias en personalidad eficiente entre los cinco grupos profesionales

Para este análisis se contó con la totalidad de la muestra. En general, los resultados obtenidos a través de un análisis de varianza simple indican que, contrariamente a lo que habíamos hipotetizado, entre los estudiantes universitarios de los diversos grupos profesionales no existen diferencias significativas en cuanto a sus características de personalidad eficiente. En la tabla 8 se muestran las medias correspondientes a los cinco grupos profesionales en los cuatro factores obtenidos (se indican las desviaciones típicas entre paréntesis). Concretamente, en el factor Personalidad eficaz, de interés más central en nuestro estudio, no se obtienen diferencias estadísticamente significativas entre los 5 grupos profesionales $(\mathrm{F}=1.163 ; \mathrm{p}=0.327)$. Igualmente, en los factores Autocontrol $y$ esfuerzo ( $\mathrm{F}=1.328 ; \mathrm{p}=0.258)$ y Asertividad $(\mathrm{F}=0.437 ; \mathrm{p}=0.782)$, las diferencias no son significativas. Las diferencias en el factor Empatía y autoestima están cercanas al nivel de significación ( $F=2.205 ; \mathrm{p}=0.067)$. 
Tabla 8. Medias y desviaciones típicas en factores de personalidad de los cinco grupos profesionales

\begin{tabular}{|l|c|c|c|c|c|}
\hline $\begin{array}{l}\text { Factores de Personalidad } \\
\text { Eficiente }\end{array}$ & Cultural & Biosanitario & Experimental & Educación & $\begin{array}{l}\text { Socio- } \\
\text { economico }\end{array}$ \\
\hline Personalidad eficaz & $54.38(8.23)$ & $56.10(5.45)$ & $54.54(7.44)$ & $55.87(7.55)$ & $54.39(7.01)$ \\
\hline Autocontrol y esfuerzo & $14.93(2.27)$ & $15.75(2.07)$ & $15.05(1.98)$ & $15.25(2.00)$ & $15.13(2.01)$ \\
\hline Empatía y autoestima & $4.61(1.23)$ & $4.75(1.08)$ & $4.57(1.22)$ & $4.26(1.25)$ & $4.50(1.20)$ \\
\hline Asertividad & $5.51(1.31)$ & $5.64(1.19)$ & $5.46(1.09)$ & $5.64(1.41)$ & $5.48(1.33)$ \\
\hline
\end{tabular}

\section{Discusión}

En la presente investigación se comprueba que la personalidad eficaz está asociada a una toma de decisiones vocacionales más madura. De un modo específico, se constata que el alumnado universitario caracterizado por la iniciativa, el optimismo, la persistencia, la tolerancia a la frustración, la adaptabilidad al medio socio-académico, la autoeficacia y la ausencia de temor al fracaso, elige su carrera basándose mucho más en factores genuinamente vocacionales, como son el conocimiento tanto de sí mismo como del mercado laboral, la confianza en los propios recursos personales y haciendo un mejor aprovechamiento de las oportunidades formativas que se vayan presentando, con lo cual los resultados obtenidos confirman las hipótesis primera y segunda.

Por otro lado, y contrariamente a lo pronosticado en nuestra hipótesis tercera, apenas hay diferencias entre el estudiantado de alta y baja personalidad eficaz en las influencias ejercidas por otras personas y circunstancias contextuales a la hora de tomar decisiones vocacionales. Igualmente, tanto los estudiantes con alta como con baja personalidad eficaz prestan igual atención a las salidas laborales y a la dificultad de las carreras universitarias, en el momento de considerarlas para su posible elección.

El factor específico de personalidad relativo al autocontrol y capacidad de esfuerzo, se comporta de un modo bastante similar al factor de personalidad eficaz, pues los estudiantes con alta puntuación en dicho factor manifiestan igualmente basar sus decisiones vocacionales más en el conocimiento de sí mismos y del mercado laboral y menos en el azar.

Contrariamente, en los otros dos factores de personalidad analizados, encontramos que los estudiantes con mayor empatía y autoestima y asertividad manifiestan tener más en cuenta el ambiente social, familiar y académico al tomar sus decisiones vocacionales.

De este conjunto de resultados obtenidos en la presente investigación podemos afirmar que las dimensiones de fortalezas, demandas y retos constitutivas del constructo psicológico de personalidad eficaz, tal y como Martín del Buey y col. (2004) lo describen, están asociadas a una toma de decisión vocacional por parte del estudiantado universitario más madura e independiente, por el contrario, la dimensión relaciones de la personalidad eficaz (empatía, asertividad) queda más vinculada a una toma de decisión vocacional más dependiente del contexto que rodea al sujeto, sea social, familiar o académico.

Las diferencias entre el estudiantado universitario de diferentes titulaciones, en cuanto a características de personalidad, no han sido confirmadas en la presente investigación, puesto que sólo en los factores de personalidad eficaz y empatía y autoestima se observa una ligera tendencia superior en el grupo sanitario, en comparación a los otros cuatro grupos profesionales, aunque no llega a ser significativa. Nosotros planteamos nuestra hipótesis avalada por otros hallazgos sobre abandono (Bethencourt y col., 2008; Cabrera y col., 2006), en los que el grupo de ciencias de la salud mostraba mayor persistencia, con puntuaciones altamente significativas en comparación con los otros grupos. 
En la investigación sobre las vinculaciones entre la personalidad y la toma de decisiones vocacionales han sido muchas las evidencias encontradas que constatan dichas relaciones. Son diversas las variables, medidas, o constructos hipotéticos de personalidad que han demostrado su conexión con la toma de decisión vocacional. En tal sentido, Saka y Gati (2007) encontraron que los estudiantes universitarios con baja autoestima, alta ansiedad como rasgo, alta indecisión general y baja identidad mostraban mayores dificultades para tomar una decisión vocacional persistente.

Por otro lado, la relación entre la autoeficacia y el compromiso con la elección vocacional en estudiantes universitarios ha sido demostrada por Wang y col. (2006).

Los intereses vocacionales son uno de los factores más influyentes en la toma de decisiones vocacionales de los estudiantes de diferentes edades, lo que explica la atención prestada a ellos en la investigación psicoeducativa, y más concretamente a las relaciones entre intereses vocacionales y personalidad. En esta línea, nos encontramos con trabajos ilustrativos como el de Sullivan y Hansen (2004), quienes demuestran que la personalidad y los intereses vocacionales mantienen claras relaciones entre si, pues la extraversión y la apertura a la experiencia, exhiben vínculos con algunos de los intereses vocacionales del modelo de Holland (1992) RIASEC (Realista, Investigador, Artístico, Social, Emprendedor, Convencional), más específicamente, la extraversión se asocia a los intereses vocacionales social y emprendedor, mientras que la apertura a la experiencia queda vinculada al interés artístico y la apertura a las ideas se relaciona con el interés investigador.

Dentro de este grupo de investigaciones centradas en el análisis de las relaciones entre personalidad e intereses vocacionales, nos encontramos también con el estudio de Mount, Barrick y Scullen (2005), que utili- zando las técnicas del análisis de cluster y del escalamiento multidimensional, obtienen que las relaciones entre personalidad e intereses vocacionales pueden ser explicadas en base a tres dimensiones de orden superior: (a) Intereses versus personalidad; (b) Orientación hacia el logro versus orientación hacia el crecimiento personal; (c) Interactuar con personas versus interactuar con cosas.

Las relaciones entre personalidad e intereses vocacionales han sido investigadas en los niveles fenotípico y genotípico por Harris y col. (2006), los cuales encontraron en gemelos y hermanos que los componentes genéticos explican hasta un 56\% de la varianza en los intereses vocacionales y un $65 \%$ en la personalidad. Tales autores afirman que la personalidad está relacionada con algunos intereses vocacionales y que algunas de esas relaciones observadas tienen una base genética común.

Concluimos, coincidiendo con Walsh y Eggerth (2005), en que el interés renovado por investigar las relaciones entre la personalidad y la toma de decisiones vocacionales viene dado en gran medida, por las implicaciones futuras que tales vínculos poseen para el sujeto, en términos de su desempeño laboral, satisfacción laboral y bienestar subjetivo.

\section{Referencias}

Aciego, R., Domínguez, R. y Hernández, P. (2005). Consistencia interna y estructura factorial de un cuestionario sobre autorrealización y crecimiento personal. Psicothema, 17(1), 134-142.

Amir, T., Gati, I. y Kleiman, T. (2008). Understanding and interpreting career decision-making difficulties. Journal of Career Assessment, 16(3), 281-309.

Bandura, A. (1997). Self-efficacy: the exercise of control. New York: Freeman.

Bethencourt, J. T., Cabrera, L., Hernández, Álvarez, P. y González, M. (2008). Variables psicológicas y educativas en el abandono universitario. Revista de Investigación Psicoeducativa, 6(3), 293-307. También on- 
line en Revista Electrónica de Investigación Psicoeducativa (http://www.investigacionpsicopedagogi-

ca.org/revista/articulos/16/espannol/Art_16 223.pdf).

Cabrera, L., Bethencourt J. T., Álvarez, P. y González, M. (2006). El problema del abandono de los estudios universitarios. $R e$ lieve: Revista Electrónica de Investigación Educativa, 12(2). http://www.uv.es/RELIEVE/v12n2/RELIE VEv12n2_1.htm

Carbonero, M. A. y Merino, E. (2004). Autoeficacia y madurez vocacional. Psicothema, 16(2), 229-234.

Cardona, C. (2002). Introducción a los métodos de investigación en educación. Madrid: EOS.

Dapelo, B. y Martín del Buey, F. (2007). Personalidad eficaz en el contexto de la educación técnico-profesional. Revista de Orientación Educacional, 39, 13-29.

Gati, I., Landman, S., Davidovitch, S., Asulin-Peretz, L. y Gadassi, R. (2010). From career decision-making styles to career decision-making profiles: A multidimensional approach. Journal of Vocational Behavior, 76(2), 277-291.

González, M. C., Álvarez, P., Cabrera, L. y Bethencourt, J. T. (2002). La toma de decisiones académicas del estudiantado de la Universidad de La Laguna en la elección de créditos de libre configuración. Contextos Educativos, 5, 123-139.

González Afonso, M.; Álvarez Pérez, P.; Cabrera Pérez, L.; y Bethencourt Benítez, J.T. (2007). El abandono de los estudios universitarios: factores determinantes y medidas preventivas. Revista Española de Pedagogía, 236, 71-85.

Holland, J. L. (1992). Making vocational choices: A theory of vocational personalities and work environments ( $2^{\text {nd }}$ ed.). Odessa: FL, Psychological Assessment Resources.

Krieshok, T. S., Black, M. D. y McKay, R. A. (2009). Career decision making: The limits of rationality and the abundance of non-conscious processes. Journal of Vocational Behavior, 75(3), 275-290.

López, M. L. y Rivas, F. (2003). Toma de decisiones vocacionales. En F. Rivas: Asesoramiento vocacional. Teoría, práctica $e$ instrumentación (377-410). Barcelona: Ariel.

Marcone, R., Martín, E., Martín del Buey, F., Dapelo, B. y Fernández, A. (2006). Adaptación chilena del cuestionario personalidad eficaz para adolescentes. Psicothema, 18(1), 130-134.

Martín Cabrera, E. y González Afonso, M. (Coord.) Rodríguez Gómez, J.M.; Pérez González, C.; Álvarez Pérez, P.; Cabrera Pérez, L.; Bethencourt Benítez, J.T.; Páez Ojeda, C.; y Palenzuela López, A. (2010). El rendimiento académico del alumnado de nuevo ingreso en la Universidad de La Laguna. Servicio de Publicaciones Universidad de La Laguna. ISBN: 978-84-7756921-3.

Martín del Buey, F., Fernández, A., Martín, E., Dapelo, B., Marcone, R. y Granados, P. (2008). Cuestionario de personalidad eficaz para la formación profesional. Psicothema, 20(2), 224-228.

Martín del Buey, F., Martín, E., Fernández, A., Dapelo, B. y Marcone, R. (2004). Evaluación de la personalidad eficaz en contextos educativos: primeros resultados. Revista de Orientación Educacional, 33-34, 79-101. Mehran, F. (2010). Psychologie positive et personnalité. Paris: Elsevier-Masson SAS.

Mount, M. K., Barrick, M. R. y Scullen, S. M. (2005). Higher-order dimensions of the big five personality traits and the big six vocational interest types. Personnel Psychology, 58(2), 447-478.

Rivas, F. (2005). Conducta vocacional y asesoramiento profesional de los estudiantes universitarios: protocolo informático para la autoayuda preprofesional. Informe final del proyecto I+D BS02001-3150, Universidad de Valencia.

Rocabert, E., Gómez, A. y Descals, A. (2006). Elaboración de un cuestionario de personalidad eficiente para el análisis de la conducta vocacional universitaria. Revista 
galego-portuguesa de psicoloxía e educación: revista de estudios e investigación en psicología y educación, 13, 399-412.

Saka, N. y Gati, I. (2007). Emotional and personality-related aspects of persistent career decision-making difficulties. Journal of Vocational Behavior, 71 (3), 340-358.

Staudinger U. M., Bowen, C. E. (2010). Life-span perspectives on positive personality development in adulthood and old age. In M. E. Lamb, A. M. Freund, y R.M. Lerner (Eds.). The handbook of life-span development, vol. 2: Social and emotional development. Hoboken, NJ, US: John Wiley \& Sons Inc. pp. 254-297.

Sullivan, B. A. y Hansen, J. C. (2004). Mapping association between interest and personality: toward a conceptual understanding of individual differences in vocational behaviour. Journal of Counseling Psychology, 51(3), 287-298.

Tian, L., Hou, Z. Shao, J. (2010). Utility of problem-solving training on career decision-making ability of university students. Chinese Journal of Clinical Psychology, 18(5), 675-679.
Walsh, W. B. (2004). Vocational psychology and personality. En B. Schneider y D. B. Smith (Eds.), Personality and Organizations (pp. 141-161). Mahwah, NJ: LEA.

Walsh, W. B. y Eggerth, D. E. (2005). Vocational psychology and personality: The relationship of the five-factor model to job performance and job satisfaction. En W. B. Walsh y M. L. Savickas (Eds.), Handbook of vocational psychology: Theory, research and practice (pp. 267-295). Mahwah, NJ: LEA.

Wang, N., Jome, L. M., Haase, R. F., Bruch, M. A. (2006). The role of personality and career decision-making self-efficacy in the career choice commitment of college students. Journal of Career Assessment, 14(3), 312-332.

\section{Notas}

[i] Las alumnas que efectuaron la aplicación de los instrumentos fueron Artemisa Barreto Espino, Piedad García Piñero, Mercedes Hernández Pedreira, Mónica Montesdeoca Bosa y Omaira Pérez García.

\section{$\underline{\text { ABOUT THE AUTHORS / SOBRE LOS AUTORES }}$}

José Tomás Bethencourt Benítez (jbethen@ull.es): profesor titular del área de Psicología Evolutiva y de la Educación. Es el autor de contacto para este artículo. Sus líneas de investigación están relacionadas con la psicología escolar. Su dirección postal es Facultad de Educación. Campus Central. Universidad de La Laguna. 38204-La Laguna. Tenerife.. Buscar otros artículos de este autor en Scholar Google

\section{Google}

Lidia Cabrera Pérez (dcabrera@ull.es): es profesora titular del área Métodos de Investigación y Diagnóstico en Educación (MIDE). Sus líneas de investigación están relacionadas con la orientación en la universidad y la evaluación e intervención socioeducativa. Su dirección postal es Departamento de Didáctica e Investigación Educativa. Facultad de Educación. Campus Central. Universidad de La Laguna. 38204-La Laguna. Tenerife. Buscar otros artículos de esta autora en Scholar Google 


\section{ARTICLE RECORD / FICHA DEL ARTÍCULO}

\begin{tabular}{|c|c|}
\hline $\begin{array}{l}\text { Reference / } \\
\text { Referencia }\end{array}$ & 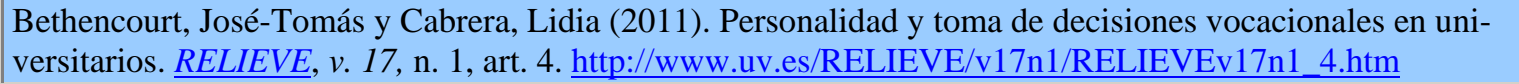 \\
\hline Title / Título & $\begin{array}{l}\text { Personalidad y toma de decisiones vocacionales en universitarios. [Personality and career decision making } \\
\text { in undergraduates]. }\end{array}$ \\
\hline $\begin{array}{l}\text { Authors / } \\
\text { Autores }\end{array}$ & Bethencourt, José-Tomás y Cabrera, Lidia \\
\hline $\begin{array}{l}\text { Review / } \\
\text { Revista }\end{array}$ & RELIEVE (Revista ELectrónica de Investigación y EValuación Educativa), v. 17, n. 1 \\
\hline ISSN & $1134-4032$ \\
\hline $\begin{array}{l}\text { Publication } \\
\text { date / } \\
\text { Fecha de } \\
\text { publicación }\end{array}$ & 2011 (Reception Date: 2010 June 23 ; Approval Date: 2011 June 26. Publication Date: 2011 June 27). \\
\hline $\begin{array}{l}\text { Abstract / } \\
\text { Resumen }\end{array}$ & $\begin{array}{l}\text { The relationships between personality and career decision making in undergraduates are analyzed in this } \\
\text { work. The hypothesis is that efficient personality is associated with the more mature process of career deci- } \\
\text { sion making. For this hypothesis, the Questionnaire of Efficient Personality and the Inventory of Career } \\
\text { Factors was administered to } 497 \text { students in their final year of undergraduate school. The collected data } \\
\text { was put under factorial analysis, analysis of differences of averages, and analysis of variance. The results } \\
\text { confirm that an effective personality is tied to career decision making based as much on one's knowledge of } \\
\text { oneself as an understanding of the working world. } \\
\text { En este trabajo se analizan las relaciones entre la personalidad y la toma de decisiones vocacionales en es- } \\
\text { tudiantes universitarios. La hipótesis de partida es que la personalidad eficaz está asociada a un proceso de } \\
\text { toma de decisiones vocacionales más maduro. Para ello se administró el Cuestionario de Personalidad Efi- } \\
\text { ciente y el Inventario de Factores Vocacionales a } 497 \text { estudiantes de último y penúltimo curso de carrera. } \\
\text { Se encontraron diferencias significativas en las dimensiones constitutivas de la decisión vocacional en gru- } \\
\text { pos extremos de alta y baja personalidad eficiente. Los resultados confirman que la personalidad eficaz está } \\
\text { vinculada a una toma de decisión vocacional más basada en el conocimiento óptimo tanto de sí mismo co- } \\
\text { mo del mercado laboral. }\end{array}$ \\
\hline $\begin{array}{l}\text { Keywords / } \\
\text { Descriptores }\end{array}$ & $\begin{array}{l}\text { Personality, Career decision making, Career development, Undergraduates. } \\
\text { Personalidad, Toma de decisiones vocacionales, Desarrollo vocacional, Estudiantado universitario. }\end{array}$ \\
\hline $\begin{array}{l}\text { Institution / } \\
\text { Institución }\end{array}$ & Facultad de Educación. Universidad de La Laguna (España). \\
\hline $\begin{array}{l}\text { Publication site } \\
\text { / Dirección }\end{array}$ & http://www.uv.es/RELIEVE \\
\hline $\begin{array}{l}\text { Language / } \\
\text { Idioma }\end{array}$ & Español (Title, abstract and keywords in English \& Spanish) \\
\hline
\end{tabular}

\section{RELIEVE}

\section{Revista ELectrónica de Investigación y EValuación Educativa E-Journal of Educational Research, Assessment and Evaluation}

[ISSN: 1134-4032]

(C) Copyright, RELIEVE. Reproduction and distribution of this articles it is authorized if the content is no modified and their origin is indicated (RELIEVE Journal, volume, number and electronic address of the document).

(C) Copyright, RELIEVE. Se autoriza la reproducción y distribución de este artículo siempre que no se modifique el contenido y se indique su origen (RELIEVE, volumen, número y dirección electrónica del documento). 